\title{
A Langevin dynamics approach to the distribution of animal move lengths
}

\author{
J. V. Santana-Filho, ${ }^{1}$ E. P. Raposo, ${ }^{1, *}$ A. M. S. Macêdo, ${ }^{1}$ G. L. \\ Vasconcelos, ${ }^{2}$ G. M. Viswanathan, ${ }^{3}$ F. Bartumeus, ${ }^{4}$ and M. G. E. da Luz ${ }^{2}$ \\ ${ }^{1}$ Laboratório de Física Teórica e Computacional, \\ Departamento de Fúsica, Universidade Federal de Pernambuco, \\ 50670-901, Recife, Pernambuco, Brazil \\ ${ }^{2}$ Departamento de Física, Universidade Federal do Paraná, \\ 81531-990, Curitiba, Paraná, Brazil \\ ${ }^{3}$ National Institute of Science and Technology of \\ Complex Systems and Departamento de Física, \\ Universidade Federal do Rio Grande do Norte, 59078-970, Natal, Brazil \\ ${ }^{4}$ Centre d'Estudis Avançats de Blanes - CEAB-CSIC, 17300, Girona, Spain
}




\begin{abstract}
Movement is fundamental to the animals ecology, determining how, when, and where an individual interacts with the environment. The animal dynamics is usually inferred from trajectory data described as a combination of moves and turns, which are generally influenced by the vast range of complex stochastic stimuli received by the individual as it moves. Here we consider a statistical physics approach to study the probability distribution of animal move lengths based on stochastic differential Langevin equations and the superstatistics formalism. We address the stochastic influence on the move lengths as a Wiener process. Two main cases are considered: one in which the statistical properties of the noise do not change along the animal's path and another with heterogeneous noise statistics. The latter is treated in a compounding statistics framework and may be related to heterogeneous landscapes. We study Langevin dynamics processes with different types of nonlinearity in the deterministic component of movement and both linear and nonlinear multiplicative stochastic processes. The move length distributions derived here comprise the possibility of movement multiscales, diffusive and superdiffusive (Lévy-like) dynamics, and include most of the distributions currently considered in the literature of animal movement, as well as some new proposals.
\end{abstract}

\footnotetext{
*E-mail: ernesto@df.ufpe.br
} 


\section{INTRODUCTION}

Movement is one of the main components of animal ecology [1-11]. The animal dynamics is essential to most major behavioral processes, from gathering food to dispersal and mating, influencing many individual vital actions and the populations survival. The statistical study of movement patterns and the mechanisms underlying movement dynamics is an invaluable source of information on the fundamental drives of animal behavior. Recently, the rise of affordable and cutting edge tracking technologies has revolutionized the study of animal behavior, particularly the field of movement ecology [1]. Notwithstanding, despite the technological advances and promise of big behavioral data [12], their inherent complexity often makes very difficult to unveil the processes controlling the animal movement dynamics [3, 4].

In this context, the development of compelling and comprehensive theoretical frameworks that advance on the study of the statistical patterns of animal movement is quite important. With this motivation, in this work we consider a statistical physics approach to the probability distributions of animal displacements, or move lengths, based on stochastic differential Langevin equations and the superstatistics formalism.

We remark that, despite the fact that many organisms present intermittent move-pause behavior (e.g., insects), and therefore display well-defined move lengths, there are many others that move about sinuously and continuously. For the latter, and considering the added complication of data gaps, there are some difficulties in extracting the move lengths from the tracking datasets $[3,13,14]$, although several methodological improvements are currently available $[1,15,16]$. In this sense, the raise of biologged and highly-resolved dataalong with theoretical advances - should definitively pave the way to segment accurately trajectories into move lengths [17].

The animal movement dynamics is intrinsically related to the distributions of move lengths and turning angles, which in theoretical works are often considered to bear little or no correlation $[5,6]$. Nevertheless, it is also important to remark that approaches exist in the literature in which move lengths and turning angles are correlated, e.g., through a movement mode with small steps/large angles alternating with long steps/small angles, such as in multiple-states random walk models of animal motion [18, 19]. Noteworthy, the way an organism processes its dynamical variability on time strongly influences its fundamental activities, being determinant to define, among others, its spreading rates and the efficiency of 
its search for resources, which can become a key issue mainly in depleted areas [5, 6, 20-23].

Part of the complexity involved in the calculation of the distribution of move lengths $\ell$ concerns the fact that a given animal displacement may result from different internal motivations, several information feedbacks from external interactions, and diverse ecological contexts (e.g., navigation, search, patch exploitation, and dispersal). Thus, unless one experimentally controls or manipulates the animal behavior in the laboratory, it becomes a rather difficult task, even for highly resolved data, to associate specific characteristics of animal behavior with some particular distribution $P(\ell)$. In other words, how to precisely justify, from a theoretical standpoint, a given distribution $P(\ell)$ (e.g., exponential, gamma, power-law, etc.), extracted from the statistical analysis of empirical data, with basis on clear-cut links to first-principle features of the animal's behavior, inner metabolism and/or interaction with the environment is a huge challenge that still remains currently unsettled.

Nevertheless, from the statistical physics point of view the problem can be simplified by considering the animal displacements as being driven by microscopic forces (e.g., local stochastic stimuli) without worrying much about their ultimate behavioral causes. In this sense, here we address the problem of calculating the distribution of move lengths $P(\ell)$ with basis on a Langevin dynamics approach $[10,24]$ and the superstatistics formalism [25, 26], which incorporate as main ingredients the degree of nonlinearity of the deterministic component of motion and the general stochastic properties driving the move length dynamics along the animal's path. We stress that our statistical physics approach does not aim to model each organism-specific microscopic mechanism driving the motion dynamics of every animal species. In the context of the discussion above, this means that we do not attempt, for instance, to establish precise links between first-principle microscopic features of the animal's behavior and the specific parameters of the mathematical functions that enter the Langevin formulation of the problem. Noteworthy, however, we also mention that, despite the wide range of inherent differences across species, the diversity of statistical movement patterns and associated animal move length distributions is not so broad [1-11].

We consider two types of motion dynamics in this work. In the first, the stochastic influence on the move lengths present steady statistical properties along the whole animal's trajectory. Moreover, in the second type we approach the more general case in which the noise statistics is heterogeneous, possibly reflecting some sort of environmental heterogeneity. We are able to generate most of the relevant move length distributions $P(\ell)$ that have 
been usually considered in the literature of movement ecology. Diffusive and Lévy-like superdiffusive movement dynamics $[5,6]$ emerge naturally in this context, as well as the possibility of multiscale movement behavior [27, 28].

Of course, the situations considered in this work do not exhaust the vast range of possibilities of animal motion dynamics. For example, our Langevin approach with Markovian temporal evolution does not contemplate continuous time random walk models of animal movement described by fractional Brownian motion dynamics [29-32], which can be associated with Lévy flights and walks, even though, from a rigorous point of view, it is not expected that the move length distributions describing animal dynamics present infinite second moment. Furthermore, the assumption that animals do not have long range memory can also be an issue in Markovian random walk models of animal motion, and appending correlations between moves can help to mitigate this problem [3]. In this sense, non-Markovian models represent an important class typically generating anomalous dynamics described, for instance, by generalized Langevin equations with memory kernels [33-36].

This work is organized as follows. In Section II we present the Langevin dynamics model of move lengths and apply it initially to the case of steady linear and nonlinear noise statistics. Next, situations with heterogeneous noise along the animal's path are considered. Final discussions and concluding remarks are left to Section III. We also include two appendices to review the connection between the Langevin dynamics and Fokker-Planck equation, and to describe the basic features of the Milstein method employed for the numerical solution of some stochastic differential Langevin equations in this work.

\section{STOCHASTIC DYNAMICAL MODEL FOR THE DISTRIBUTION OF ANI- MAL MOVE LENGTHS}

Move lengths represent bouts of motion, the summation of a number of move steps with relatively constant direction. We begin by considering a given move length that starts at time $t=0$ and ends at $t=t_{f}$. Let us define the variable $\ell(t)$ as the distance from the starting point of the move to the individual's current position at a time $t \leq t_{f}$. Thus, one has $\ell(0)=0$ and the length of this particular move corresponds to $\ell\left(t_{f}\right)$. In the sequence, as this move comes to an end, the animal embarks on a new move along a new direction and possibly with a distinct duration $t_{f}^{\prime}$. We thus reset the time again to $t=0$ at the beginning 
of the new move, which therefore finishes with length $\ell\left(t_{f}^{\prime}\right)$. As this process is repeated oftentimes, a sequence of $N$ move lengths $\left\{\ell_{j}\right\}$ is generated (an ensemble of $N$ realizations of this process), with $j=1,2, \ldots, N$.

Here we present a stochastic dynamical model for the variable $\ell(t)$ from which each move length is determined. We shall assume that the specific duration $t_{f}$ of each move is much larger than any characteristic time involved in the dynamics of $\ell(t)$. Thus, for all practical purposes the distribution of a number $N \gg 1$ of move lengths in the ensemble $\left\{\ell_{j}\right\}$ shall correspond to the probability distribution $P(\ell)$ resulting from the $t \rightarrow \infty$ stationary regime of the dynamical model for $\ell(t)$.

In the stochastic modeling of animal movement, one possibility is to ideally seek to describe the individual's trajectory in space in terms of a Langevin dynamics, such as a Brownian motion or the Ornstein-Uhlenbeck process or variations thereof [6]. However, the extension of such processes to two or three dimensions, although in principle mathematically simple, can introduce some extra difficulties [6]. For instance, the fact that in two-dimensional Brownian motion the dynamics in the $x$ and $y$ directions are mutually independent is not, in many practical cases, a reasonable hypothesis. In this sense, introducing correlation brings in additional mathematical complications. Other possible ways to circumvent this type of problem, such as in approaches based on turn-angle distributions [3], have their own limitations [6]. Here we shall instead develop an effective model to describe the fluctuations in the move length $\ell$ itself. More specifically, our goal is to deduce the probability distribution $P(\ell)$ without having to resolve the details of the animal's trajectory. In other words, rather than attempting to model the trajectories (from which one could eventually deduce the move length distribution), we shall instead propose a stochastic dynamics directly for $\ell$ and investigate which classes of such models are relevant, with the calculation of their corresponding distributions. As we will see below, our approach has the advantage that it combines mathematical simplicity and statistical generality.

In formulating our model, we consider that the animal motion, and hence the variable $\ell(t)$, results from the interplay of both deterministic and stochastic components. More concretely, we shall formulate the model in terms of a stochastic differential equation (SDE) expressing the general Langevin dynamics of $\ell(t)$,

$$
d \ell(t)=F(\ell) d t+G(\ell) d W_{t}
$$


where the function $F(\ell)$ setting the drift-like deterministic part of the model in principle can be nonlinear. Also, we assume that the diffusion-related noise term is described by a Wiener process $d W_{t}=\xi(t) d t$ modulated by the general function $G(\ell)$, where $\xi(t)$ has Gaussian distribution (Gaussian white noise), with mean $\langle\xi(t)\rangle=0$ and correlation $\left\langle\xi(t) \xi\left(t^{\prime}\right)\right\rangle=$ $2 \varepsilon \delta\left(t-t^{\prime}\right)$, and $\delta$ denotes the Dirac delta function. We therefore notice that the parameter $\varepsilon>0$ gives the magnitude of noise fluctuations ( $2 \varepsilon$ is the variance of the Gaussian). This stochastic contribution can be thought of as coming from a randomly fluctuating (noise) term related to the broad range of unpredictable stimuli generally received by the individual as it moves. We also note that $F(\ell)$ in (1) plays the role of an effective velocity that the animal would have in the absence of random noise. Owing to the occurrence of such noise, the animal then adjusts its speed accordingly, which is reflected in the noise amplitude $G(\ell)$.

The dynamics of the variable $\ell(t)$ is fully determined in the Langevin equation (1) by the functions $F(\ell)$ and $G(\ell)$ and the noise magnitude $\varepsilon$. Therefore, in the specific context of animal motion it becomes necessary to impose a number of associated constraints on the functions $F(\ell)$ and $G(\ell)$ that we discuss in the following.

First, we observe that $\ell(t)$ is a positive definite variable, i.e., $\ell(t) \geq 0$ for all $t \geq 0$, since it gives the individual's distance at time $t$ to the starting point of the move. By hypothesis, $\ell(t)$ is driven by a continuous stochastic process, as it is described by the Langevin dynamics (1). Thus, if a given realization of the process (1), starting from $\ell(0) \geq 0$, were to give a negative value of $\ell$ at some time $t^{\prime}$, then it would necessarily have reached the value zero at some earlier time $t<t^{\prime}$, implying $\ell(t)=0$. Thus, by setting $\ell(t)=0$ in Eq. (1), we get $d \ell(t)=F(0) d t+G(0) d W_{t}$. If we furthermore impose that $F(0)>0$ and $G(0)=0$, then $d \ell(t)>0$, from which we conclude that $\ell$ could not have become negative, hence $\ell \geq 0$ for the whole process. In other words, the conditions $F(0)>0$ and $G(0)=0$ are sufficient to ensure that $\ell$ stays positive if $\ell(0) \geq 0$. In the argument above, we observe that it was crucial that the noise amplitude vanishes for $\ell=0$, i.e., $G(0)=0$. In particular, this constraint excludes several possibilities for the stochastic term, one of them being the additive noise, $G(\ell)=$ constant. As a consequence,in this work we study the action of an $\ell$-dependent multiplicative noise [24] on the dynamics of $\ell(t)$, including some linear and nonlinear forms for $G(\ell)$. An analogous situation also emerges in the context of macroscopic theories of stochastic population models, where the noise must be multiplicative in order to avoid negative values of the population density [37-39]. We further comment that a positive 
definite noise term, such as the one described by a Poisson distribution, could also prevent negative move lengths, although we have not been able to obtain analytical expressions for $P(\ell)$ in this case. Indeed, the introduction of stochastic processes of this type may lead to additional complications, such as, for instance, the corresponding Fokker-Planck equation becomes an integro-differential equation [40,41], which we believe is not justifiable nor necessary for our goals here.

In addition, we remark in the context of the distribution of animal move lengths that $P(\ell \rightarrow \infty)=0$ should arise as a natural constraint, as well as the finiteness of the second moment of $P(\ell)$ due to either environmental and/or behavioral restrictions in the realistic animal motion dynamics. For instance, we notice in the case of linear multiplicative noise, $G(\ell)=\ell$, that rather large values of $\ell$ can be hampered in Eq. (1) by imposing $F(\ell \rightarrow \infty)=-\infty$, so to yield a strongly negative $d \ell / d t$ for large $\ell$. This feature impacts directly on the degree of diffusivity of the animal trajectory, as discussed below.

\section{Homogeneous noise statistics}

We begin by considering the situation in which the noise magnitude $\varepsilon$ is constant along the animal's path. This means that the Gaussian distribution of the noise variable $\xi$, with null mean and variance $2 \varepsilon$, is the same for each move. In this case, one can think of an animal in an environment roughly steady in time and spatially homogeneous (environments with high spatial heterogeneity and/or rapidly changing in time will be addressed below).

Before turning to the analysis of some particular choices of functions $F(\ell)$ and $G(\ell)$, we briefly discuss the general role of the initial condition on the Langevin dynamics, Eq. (1). At $t=0$ one has $\ell(0)=0$ and $d \ell=F(\ell=0) d t$, since $G(\ell=0)=0$ as argued above. It is

thus necessary that $F(\ell=0)>0$ to yield nonzero (positive) values of $\ell(t)$ for $t>0$. Here we accomplish this by considering $F(\ell=0)=c>0$ in two situations: $c \rightarrow 0^{+}$and $c \neq 0$. The families of distributions $P(\ell)$ that arise in each case are discussed below on the light of the move length distributions mostly considered in the literature of movement ecology.

\section{A. Linear noise}

The simplest choice in this case corresponds to the linear function $F(\ell)=c+a \ell$ along with linear multiplicative noise, $G(\ell)=\ell$, where $a$ is a constant and $c>0$. The probability distribution of move lengths $P(\ell)$ can be obtained from the stationary solution of the Fokker-Planck equation associated with Eq. (1) (see Appendix A). We notice, however, 


\begin{tabular}{|l|c|c|}
\hline \multicolumn{1}{|c|}{ Distribution } & Mathematical expression & Parameters \\
\hline 1. Exponential & $P(\ell)=\frac{b}{\varepsilon} \exp (-b \ell / \varepsilon)$ & $\gamma=1, b>0, a / \varepsilon=2$ \\
\hline 2. Gaussian (half-normal) & $P(\ell)=\sqrt{\frac{2 b}{\pi \varepsilon}} \exp \left[-b \ell^{2} /(2 \varepsilon)\right]$ & $\gamma=2, b>0, a / \varepsilon=2$ \\
\hline 3. Stretched exp (Weibull) & $P(\ell)=\frac{b}{\varepsilon} \frac{\exp \left[-b \ell^{\gamma} /(\gamma \varepsilon)\right]}{\ell^{1-\gamma}}$ & $0<\gamma \neq 1, b>0, a / \varepsilon=1+\gamma$ \\
\hline 4. Gamma & $P(\ell)=\mathcal{A} \frac{\exp (-b \ell / \varepsilon)}{\ell^{2-a / \varepsilon}}$ & $\gamma=1, b>0, a / \varepsilon>1$ \\
\hline 5. Inverse-gamma & $P(\ell)=\mathcal{A} \frac{\exp [-|b| /(\varepsilon \ell)]}{\ell^{2-a} / \varepsilon}$ & $\gamma=-1, b<0, a / \varepsilon<-1$ \\
\hline 6. Generalized gamma & $P(\ell)=\mathcal{A} \frac{\exp \left[-b \ell^{\gamma} /(\gamma \varepsilon)\right]}{\ell^{2-a / \varepsilon}}$ & $\gamma>0, b>0, a / \varepsilon>1$ \\
\hline 7. Inverse-to-a-power gamma & $P(\ell)=\mathcal{A} \frac{\exp \left[-b \ell^{-|\gamma|} /(\gamma \varepsilon)\right]}{\ell^{2-a / \varepsilon}}$ & $-1<\gamma<0, b<0, a / \varepsilon<-1$ \\
\hline 8. Chi-square & $P(\ell)=\frac{1}{2^{k / 2} \Gamma(k / 2)} \frac{\exp (-\ell / 2)}{\ell^{1-k / 2}}$ & $\gamma=1, \varepsilon / b=2, a / \varepsilon=1+k / 2, k=1,2 \ldots$ \\
\hline 9. Generalized Rayleigh & $P(\ell)=\left(\frac{b}{2 \varepsilon}\right)^{k / 2} \frac{2}{\Gamma(k / 2)} \frac{\exp \left[-b \ell^{2} /(2 \varepsilon)\right]}{\ell^{1-k}}$ & $\gamma=2, b>0, a / \varepsilon=1+k, k=1,2 \ldots$ \\
\hline 10. Power-law exp attenuated & $P(\ell)=\overline{\mathcal{A}} \frac{\exp \left[-b \ell^{\gamma} /(\gamma \varepsilon)\right]}{\left(\ell+\ell_{0}\right)^{n / \varepsilon} \ell^{2-a / \varepsilon}}$ & $\gamma>0, b>0$, see text for $a, \varepsilon, n$ \\
\hline 11. Stretched GIG & $P(\ell)=\mathcal{A} \frac{\exp \left[-\alpha_{x} \ell^{x}-\alpha_{y} / \ell^{y}\right]}{\ell^{1-p}}$ & $\alpha_{x}>0, \alpha_{y}>0 ;$ GIG: $\alpha_{x}=1, \alpha_{y}=1$ \\
\hline 12. Beta & $P(\ell)=\mathcal{C}\left(\frac{\ell}{\ell_{\max }}\right)^{\delta-1}\left(1-\frac{\ell}{\ell_{\max }}\right)^{\beta-1}$ & $\ell \leq \ell_{\max }$, see text for $\delta$ and $\beta$ \\
\hline 13. K-distribution & $P(\ell)=\overline{\mathcal{N}} \ell^{\omega} K_{\omega-1}(\bar{b} \ell)$ & $\gamma=2, b>0, \omega>0, \bar{b}^{2}=2 b \omega>0$ \\
\hline 14. Hyper-exponential & $P(\ell)=\sum_{i=1}^{s} \frac{\omega_{i}}{\lambda_{i}} e^{-\ell / \lambda_{i}}$ & $\lambda_{i}>0, \omega_{i}>0, \sum_{i=1}^{s} \omega_{i}=1$ \\
\hline
\end{tabular}

TABLE I. Summary of some distributions of move lengths arising from the general Langevin dynamics (1). In expressions 1-12 the statistics of the noise is homogeneous along the whole animal's path (in particular, in 1-10 we used Eq. (2) with $c \rightarrow 0^{+}$), while in 13-14 heterogeneous noise statistics was considered, which may be related to some degree of heterogeneity in the landscape properties. The right column indicates the possible range of the associated parameters.

that when $c \rightarrow 0^{+}$the solution obtained, $P(\ell)=\ell^{-2+a / \varepsilon}$, is not normalizable to unit, since $\int_{0}^{\infty} P(\ell) d \ell \rightarrow \infty$.

To circumvent this difficulty, we introduce some degree of nonlinearity in the deterministic term of Eq. (1), which we write in the convenient form

$$
F(\ell)=c+a \ell-b \ell^{1+\gamma}
$$

with $c>0$. The parameters $a$ and $b \neq 0$ can assume positive or negative values (see discussion on their constraints below), and for convenience we consider a properly normalized $\ell$ so that $a$ and $b$ are both dimensionless. We further take $\gamma \geq-1$ to avoid singularities in $F$, with $\gamma \neq 0$. We comment that the nonlinear feature appended in the $b$-term of Eq. (2) might 
be realistically desirable since, besides being subject to random noise, the animal movement dynamics shares many features of complex systems phenomena, which are usually nonlinear.

We first consider Eq. (2) with $c \rightarrow 0^{+}$in the Langevin dynamics (1) with linear noise. The stationary solution of the associated Fokker-Planck equation yields the normalized distribution (see Appendix A)

$$
P(\ell)=\mathcal{A} \frac{\exp \left[-b \ell^{\gamma} /(\gamma \varepsilon)\right]}{\ell^{2-a / \varepsilon}},
$$

with normalization constant $\mathcal{A}=|\gamma|(b / \gamma \varepsilon)^{(a / \varepsilon-1) / \gamma} / \Gamma[(a / \varepsilon-1) / \gamma]$ and $\Gamma$ as the gamma function. We notice that the values of the parameters in (3) cannot be fully arbitrary. For example, since $\varepsilon>0$ a real-valued distribution demands $b / \gamma>0$. Moreover, due to the gamma function the normalization condition is assured only if $(a / \varepsilon-1) / \gamma>0$. Finally, if one further requires the realistic constraint of finite second moment of the move length distribution, then $(a / \varepsilon+1) / \gamma>0$.

Equation (3) is general enough to comprise diverse sorts of distributions of move lengths, a fraction of them is displayed in the expressions 1-9 in Table I, along with the corresponding range of parameters. We observe that among these expressions one can find some of the most relevant distributions considered in the literature of movement ecology [1-6]. For example, exponential and Gaussian distributions have been widely used in the context of classic random walk models of animal motion, including correlated random walks and biased random walks $[3,42]$. Exponentials have been also applied to explain the movement patterns of several species of marine predators in waters plentiful of resources, while in depleted regions truncated power-law distributions arise [43, 44]. Furthermore, hyper-exponentials have been considered in the study of the movement of mussels [28] and bumblebees [45], while power-law distributions have been reported in the motion dynamics of spider monkeys [46] and the nematode C. elegans [47]. On the other hand, stretched exponential (Weibull) distributions emerge in the motion patterns of species as diverse as bees [45] and elks [18]. Also, gamma [48] and power-law [20] distributions have been used to describe move length datasets of wandering albatrosses.

We now turn to the general discussion of the model parameters in the context of the animals' move length data analysis. We first comment that the parameters that define the functions $F(\ell)$ and $G(\ell)$ are organism specific in the sense they reflect drives of motion dynamics which are particular of each animal species. Nevertheless, as mentioned in the 
Introduction, here we are mostly concerned with a statistical physics approach to this problem, without worrying much about the detailed organism-specific mechanisms that set the particular values of the model parameters of each individual. In this sense, it is important to remark as well that, despite the broad range of intrinsic differences between animal species, the statistical diversity of motion patterns and move length distributions is not so wide $[1-11]$.

It is first interesting to notice that the distribution $P(\ell)$ in Eq. (3) is determined by only three independent parameters: $\bar{a}=a / \varepsilon, \bar{b}=b / \varepsilon$, and $\gamma$. In other words, the magnitude $\varepsilon$ of the noise fluctuations acts in practice as a normalization factor to the coefficients $a$ and $b$ of the deterministic term defined in (2) for $c \rightarrow 0^{+}$. Moreover, the characteristic scale of $P(\ell)$ is given from Eq. (3) by $\bar{\ell}=(\gamma / \bar{b})^{1 / \gamma}$, which is a quantity that can be statistically inferred from the empirical data sets of animal move lengths. Consistently, we note that $\bar{\ell}$ depends only on the noise statistics $\varepsilon$ and the nonlinear part of the deterministic component set by $b$ and $\gamma$ (we recall above that a scale-free power-law function is obtained for $c \rightarrow 0^{+}$in the absence of nonlinearity).

On the other hand, the shape of the distribution $P(\ell)$ is driven by both deterministic and stochastic components. Interestingly, whereas both act together to govern the power-law dependence in Eq. (3) via the parameter $\bar{a}=a / \varepsilon$, the shape of the exponential attenuation of $P(\ell)$, which is the ultimate responsible for the finiteness of the second moment of the distribution, is driven as a function of $\ell / \bar{\ell}$ only by the nonlinear exponent $\gamma$. This noteworthy splitting of parameters' actions can be properly captured through the statistical analysis of the empirical move length datasets using, e.g., maximum likelihood estimation (MLE) and Akaike information criterion (AIC) methods [3, 6]. In particular, the set of best-fit parameters obtained by applying such statistical estimators to actual movement data can provide relevant information on the type and strength of nonlinearity associated with the deterministic component of movement and the general stochastic properties influencing the animal's move lengths.

We display in Fig. 1 the particular cases of gamma and inverse-gamma distributions of move lengths (see Table I). In each case a nice agreement is found between the stationary solution $P(\ell)$, Eq. (3), and the numerical simulation results of the corresponding Langevin dynamics (1), obtained by applying the Milstein method [49] (see Appendix B for details). We notice that after $N_{t}=10^{6}$ iterations of the Misltein procedure using a small time 


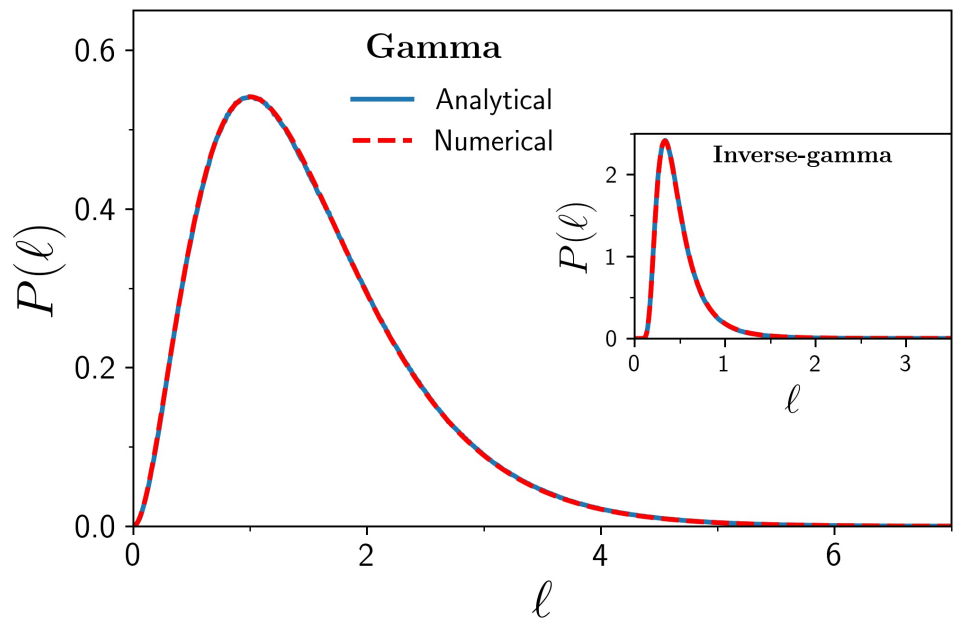

FIG. 1. Comparison between the move length distribution $P(\ell)$, Eq. (3), and the numerical simulation results from the Langevin dynamics (1) using the Milstein method. The gamma distribution (expression 4 in Table I) has parameters $\gamma=1.0, a=2.0, b=1.0$, and $\varepsilon=0.5$. Inset: inversegamma distribution (expression 5 in Table I), with $\gamma=-1.0, a=-2.0, b=-1.0$, and $\varepsilon=0.5$. An ensemble of $10^{8}$ moves was considered in each plot.

interval $\Delta t=h=10^{-3}$, i.e., after a time $t=N_{t} h=10^{3}$, the stationary regime of the Langevin dynamics (1) is fairly reached, so to yield one move length value $\ell$. An ensemble of $10^{8}$ moves was considered in each plot of Fig. 1.

We now discuss the movement dynamics of an animal with move length distribution given by Eq. (3). Since the second moment of $P(\ell)$ is finite, the central limit theorem (CLT) [5, 6] assures that the distribution of the sum of $n$ move lengths converges to a Gaussian for sufficiently large $n$. In this case, the movement dynamics is diffusive $[5,6]$, which means that the animal's rms distance after $N$ moves, or after time $t$, behaves $[5,6]$ after some initial transient period as $x_{\mathrm{rms}} \sim N^{\nu} \sim t^{\nu}$, where $\nu=1 / 2$ is the diffusion (Hurst) exponent.

On the other hand, from a statistical point of view a superdiffusive (anomalous) dynamics of a random walk particle with move length distribution $P(\ell)$, displaying Hurst exponent $\nu>1 / 2$, can also emerge in the context of the generalized CLT $[5,6]$. In this case, $P(\ell)$ has infinite second moment and the sum of $n$ move lengths converges to the family of Lévy $\alpha$-stable distributions for sufficiently large $n[5,6]$. The Lévy index lies in the range $0<\alpha \leq 2$, with the limit value $\alpha=2$ recovering the Gaussian distribution and CLT result. To illustrate the generalized CLT, consider, e.g., a random walker with move length 
distribution presenting asymptotic large- $\ell$ heavy-tail in the power-law form, $P(\ell) \sim \ell^{-\mu}$. If the power-law exponent $\mu$ lies in the range $1<\mu<3$, the distribution is normalizable and its second moment is infinite, so that the sum of $n$ move lengths approaches [5, 6] a Lévy distribution with index $\alpha=\mu-1$. Conversely, for $\mu>3$ the finite second moment yields the movement dynamics to be governed by the CLT (the borderline case $\mu=3$, corresponding to the $\alpha=2$ Gaussian limit, is also driven by the CLT) [5, 6].

Importantly, in realistic animal movement dynamics the second moment of $P(\ell)$ can be very large but must remain finite, since a limitation to the maximum move length always exists, either due to environmental and/or biological/behavioral constraints. Nevertheless, this is not to mean that superdiffusive regimes are not observed in animal movement. On the contrary, it is now well established that, under specific conditions (e.g., low resources availability), superdiffusive motion is indeed evidenced from the analysis of actual movement data of a diverse range of animal species [5, 6, 43, 44, 46, 50, 51]. However, it is important to make clear that superdiffusive behavior in realistic animal movement has an ecological scale limit. In fact, identifying the ecological range of scales where superdiffusive regimes emerge can help to understand relevant components of animal ecology [27]. In this sense, as the second moment of $P(\ell)$ is finite in animal movement behavior, superdiffusive Lévy-like dynamics cannot extend indefinitely since, according to the CLT, the convergence to the normal (Gaussian) dynamics eventually takes place, even if it occurs only rather slowly [52]. This is what we term limited superdiffusive dynamics below.

The easiest way to generate limited superdiffusive dynamics is by truncating the powerlaw distribution $P(\ell) \sim \ell^{-\mu}$ at a large upper cutoff length $\ell_{\max }$, so that $P(\ell)=0$ if $\ell>\ell_{\max }$, or by attenuating it exponentially in the large- $\ell$ limit, $P(\ell) \sim \ell^{-\mu} \exp (-\lambda \ell)$, with small $\lambda>0$. In both cases, an animal motion related to such $P(\ell)$ displays superdiffusive Lévylike dynamics to a large extent if $1<\mu<3$, with eventual convergence to the CLT for large number of moves, as mentioned.

Noteworthy, we remark that Lévy-like limited superdiffusive dynamics is actually possible in our Langevin framework. By adding to the function $F(\ell)$ in Eq. (2) with $c \rightarrow 0^{+}$the term $-v \ell^{2} /\left(\ell+\ell_{0}\right), \ell_{0}>0$, that reduces to Eq. (2) if $v=0$ and interpolates in the case $v \neq 0$ between the quadratic and linear behaviors for low- and high- $\ell$, respectively, we obtain

$$
P(\ell)=\overline{\mathcal{A}} \frac{\exp \left[-b \ell^{\gamma} /(\gamma \varepsilon)\right]}{\left(\ell+\ell_{0}\right)^{v / \varepsilon} \ell^{2-a / \varepsilon}},
$$



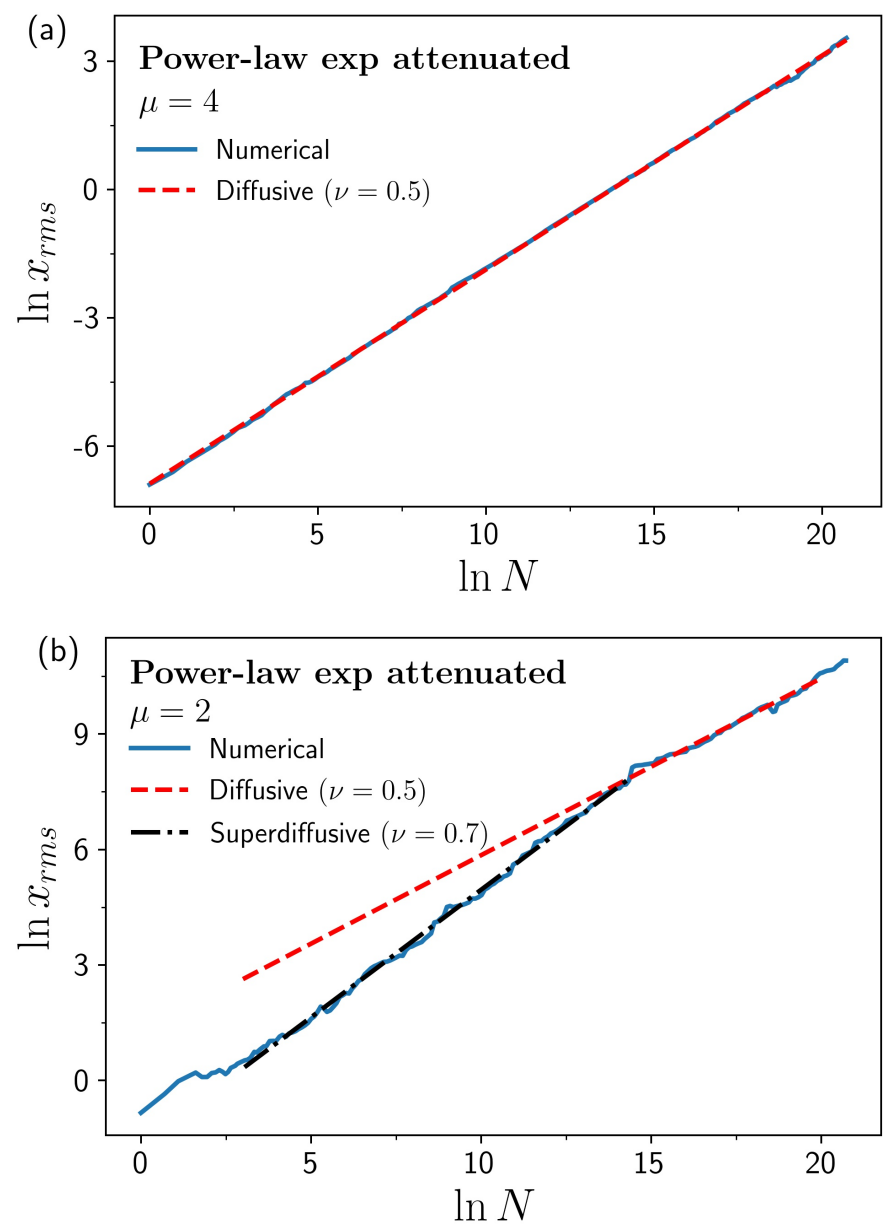

FIG. 2. Movement dynamics of a random walk particle with distribution $P(\ell)$ of move lengths displaying exponentially-attenuated power-law form, Eq. (4). For large- $\ell$, one has that $P(\ell) \sim \ell^{-\mu} \exp \left(-\lambda \ell^{\gamma}\right)$, with power-law exponent $\mu=2-(a-v) / \varepsilon$ and $\lambda=b /(\gamma \varepsilon)$. Numerical results were obtained from the simulation of the Langevin dynamics (1) using the Milstein method. The walker's rms distance $x_{\mathrm{rms}} \sim N^{\nu}$ is shown as a function of the number of moves $N$, where $\nu$ is the diffusion (Hurst) exponent. (a) For $\mu=4$ the movement dynamics is diffusive from the very beginning, as indicated by the best-fit value $\nu=0.5$. (b) In contrast, for $\mu=2$ belonging to the superdiffusive range $1<\mu<3$, a superdiffusive dynamics with $\nu=0.7$ initially arises after a transient period and remains for nearly five decades, before crossing over to the diffusive dynamics with $\nu=0.5$, as predicted by the central limit theorem (CLT). Parameters were $\gamma=0.6, a=2.0$, $b=10^{-4}, c \rightarrow 0^{+}, \varepsilon=1.0$, and $\ell_{0}=1.0$, so that $\mu=v$ and $\lambda=1.7 \times 10^{-4}$. 
where $\overline{\mathcal{A}}$ is the normalization constant. Its large- $\ell$ asymptotic limit displays the exponentiallyattenuated power-law form discussed above, with the power-law exponent $\mu=2-(a-v) / \varepsilon$. In particular, when $1<\mu<3$ Lévy-like limited superdiffusive dynamics arises from distribution (4) for $\gamma>0, b>0$, and $\varepsilon<a<v+\varepsilon$ if $0<v / \varepsilon<2$, or $v-\varepsilon<a<v+\varepsilon$ if $v / \varepsilon \geq 2$. (We note in passing that forcing the power-law exponent $\mu=2-a / \varepsilon$ in Eq. (3) to lie in the superdiffusive range would conflict with either the normalization condition or the finite second moment constraint, implying that $P(\ell)$ in $(3)$ actually gives rise only to diffusive dynamics.)

The limited superdiffusive behavior resulting from the exponentially-attenuated powerlaw $P(\ell)$, Eq. (4), can be clearly observed from the numerical simulation results shown in Fig. 2, obtained by applying the Milstein method to the Langevin dynamics (1). In this case, the choice of parameters was such that $P(\ell) \sim \ell^{-\mu} \exp \left(-\lambda \ell^{\gamma}\right)$ at large- $\ell$, with $\gamma=0.6$, $\lambda=1.7 \times 10^{-4}$, and $\mu=v$ (see caption of Fig. 2). In the numerical simulation, a random walker with move lengths drawn from this $P(\ell)$ starts from the origin in the one-dimensional space and has position after $N$ moves given by $x_{N}=\sum_{j=1}^{N} u_{j} \ell_{j}$, where the variable $u_{j}=+1$ $\left(u_{j}=-1\right)$ is randomly chosen with probability $1 / 2$ so to yield a move to the right (left). Figure 2 displays the walker's rms distance as a function of $N$. In Fig. 2 (a) we set $\mu=v=4$ in Eq. (4), therefore out of the superdiffusive range $1<\mu<3$. Consistently, upon averaging over $10^{4}-10^{6}$ walk realizations (see Appendix B), a diffusive dynamics with numerical Hurst exponent $\nu=0.5$ soon establishes and remains as such for the whole range of $N$.

In contrast, a rather different movement dynamics emerges in Fig. 2(b) when $\mu=v=2$ and $\gamma=0.6$. Now, after an initial transient period, Lévy-like superdiffusive behavior with $\nu=0.7$ is clearly identified along nearly five decades, $2.0 \times 10^{1} \lesssim N \lesssim 1.2 \times 10^{6}$, before it crosses over to the diffusive regime with $\nu=0.5$ for $N \gtrsim 1.2 \times 10^{6}$. We have also found that the diffusion exponent $\nu$ increases slowly as $\gamma$ decreases, as expected, though the $\nu=1$ ballistic limit [5, 6], obtained for a Lévy-like random walker with $\gamma \rightarrow 0$ and Lévyattracted move length distribution $P(\ell) \sim \ell^{-\mu}$ with $\mu=2$ and Lévy index $\alpha=\mu-1=1$, is prohibitively time consuming in our simulation.

We further notice, by making $\ell \rightarrow \ell-\ell_{\min }$ in Eq. (1) and expressions above for $P(\ell)$, that distributions with minimum move length $\ell_{\min }$ can be also obtained in our approach. Such distributions are relatively common in the statistical modeling of actual animal motion (for example, a gamma distribution with lower cutoff move length was applied to model the 
movement of wandering albatrosses in the wild [48], and exponential, stretched exponential, and truncated power-law distributions with minimum length were analyzed in the study of bumblebees moving in a controlled experiment [45]).

As a final remark on the case with linear noise, we now consider a positive $c \neq 0$ in the function $F(\ell)$, Eq. (2), yielding

$$
P(\ell)=\mathcal{A}^{\prime} \frac{\exp \left[-b \ell^{\gamma} /(\gamma \varepsilon)-c / \ell\right]}{\ell^{2-a / \varepsilon}} .
$$

In particular, for $\gamma=1$ (quadratic function $F$ ) $P(\ell)$ has the form of a generalized inverse Gaussian (GIG) distribution [53]. To our knowledge, the stretched GIG distribution (5) has not been applied so far in the context of animal motion dynamics, even though it is clear that in the limit $c \rightarrow 0^{+}$it recovers the commonly-used distributions generally expressed by Eq. (3) (see also Table I). A noticeable characteristic of the GIG distribution (5) is that null move lengths present null probability, i.e., $P(\ell=0)=0$, due to the exponential factor $e^{-c / \ell}$. This property may figure as a relevant constraint in statistical models of animal motion.

\section{B. Nonlinear noise}

Nonlinear noise functions $G(\ell)$ can be also considered in our framework, provided that $G(\ell=0)=0$, as argued. One relevant case in the context of animal motion concerns distributions with maximum move length $\ell_{\max }$ (upper natural length scale). For instance, by choosing in Eq. (1) $F(\ell)=a\left(\ell_{\max }-\ell\right)-b$, with $b>0$ and $a \ell_{\max }-b>0$, and $G(\ell)=\left[\ell\left(\ell_{\max }-\ell\right)\right]^{1 / 2}$, we obtain the beta distribution $[54]$

$$
P(\ell)=\mathcal{C}\left(\frac{\ell}{\ell_{\max }}\right)^{\delta-1}\left(1-\frac{\ell}{\ell_{\max }}\right)^{\beta-1}
$$

with $\mathcal{C}=\Gamma(\delta+\beta) /\left[\ell_{\max } \Gamma(\delta) \Gamma(\beta)\right], \delta=b /\left(\varepsilon \ell_{\max }\right)>0, \beta=\left(a-b / \ell_{\max }\right) / \varepsilon>0$, and move lengths limited from above, $\ell \leq \ell_{\max }$ (see Table I).

We can also generalize the linear noise of previous subsection by considering $G(\ell)=\ell^{s}$, where $s>0$. In the case $c \rightarrow 0^{+}$in Eq. (2) we obtain the general expression

$$
P(\ell)=\mathcal{C}^{\prime} \ell^{-2 s} \exp \left[\frac{a}{2 \varepsilon(1-s)} \ell^{2(1-s)}-\frac{b}{\varepsilon[2(1-s)+\gamma]} \ell^{2(1-s)+\gamma}\right],
$$

with $s \neq 1, \gamma \neq 2(s-1)$, and parameter constraints generally depending on $s$. For example, for $1<s<3 / 2$ the constraints of normalization to unity, $P(\ell \rightarrow \infty)=0$, and finite second 
moment of $P(\ell)$ imply $a>0, b>0$, and $\gamma>2(s-1)$. This case represents a sort of GIG distribution but with both exponential dependencies with compressed/stretched form, since $2(1-s)<0$ and $2(1-s)+\gamma>0$ (see Table I).

\section{Heterogeneous noise statistics}

In a long path, or in large ecological scales, the assumption made above of constant noise magnitude $\varepsilon$ might appear too restrictive in some instances. Examples include situations in which the environment properties change relatively quick, in a typical time scale that is small if compared with the largest scale $\tau$ of relevance to the movement dynamics, or when a spatially heterogeneous landscape is partitioned into patchy regions with much distinct features. In these cases, the animal possibly experiences changes in the statistical properties of the noise fluctuations as it moves. In fact, lately there has been a growing uptake of the view $[47,55]$ that the deterministic and stochastic components of the interaction with the environment and organism's behavioral drives can lead in some circumstances to move lengths that should not be strictly considered as drawn by a unique distribution along the whole animal path, possibly including $P(\ell)$ expressed even by a statistical mixture of distributions. Thus, in the following we allow general fluctuations in $\varepsilon$ so that $\varepsilon \rightarrow \varepsilon(t)$.

We start by assuming that the dynamics of the noise magnitude $\varepsilon(t)$ is characterized by some typical scale $\tau_{\varepsilon} \ll \tau$. In other words, we consider that $\varepsilon$ remains roughly constant along moves performed within the scale $\tau_{\varepsilon}$. These moves allow to build the conditional distribution $P(\ell \mid \varepsilon)$. Therefore, in order to obtain the distribution $P(\ell)$ of move lengths along the whole animal's path it is necessary to weight $P(\ell \mid \varepsilon)$ by the distribution $f(\varepsilon)$ of values of $\varepsilon$, leading to the statistics superposition integral,

$$
P(\ell)=\int_{0}^{\infty} P(\ell \mid \varepsilon) f(\varepsilon) d \varepsilon .
$$

This approach is known in other contexts as superstatistics [25, 26] (see also [56, 57] for a multiscale extension of Eq. (8)). Note that if only one given value $\varepsilon=\varepsilon^{*}$ occurs, then $f(\varepsilon)=\delta\left(\varepsilon-\varepsilon^{*}\right)$ and the previous results with constant $\varepsilon$ are recovered. In fact, by compounding two distributions in (8) one is naturally given a variety of possible expressions for $P(\ell)$ much wider than in the previous case.

The dynamics of the noise magnitude $\varepsilon(t)$ can be also formulated in terms of a Langevin equation. In this sense, we illustrate below with one relevant example of $P(\ell)$ obtained 
through the superposition integral (8). (A more detailed study of compound models will be left for future work.)

We consider the example of a compounding distribution in which the conditional distribution $P(\ell \mid \varepsilon)$ is a stretched exponential (Weibull), with fixed ratio $a / \varepsilon=1+\gamma$ (see Table I). If the distribution of values of $\varepsilon$ along the path is given by a gamma, then $f(\varepsilon)=\mathcal{N} \varepsilon^{\omega-1} \exp (-\bar{\omega} \varepsilon)$, where $\mathcal{N}$ is the normalization constant and $\omega$ and $\bar{\omega}$ are the shape and rate parameters, respectively. Thus, by performing the integral (8) we obtain

$$
P(\ell)=\overline{\mathcal{N}} \frac{K_{\omega-1}\left(\bar{b} \ell^{\gamma / 2}\right)}{\ell^{1-\gamma(\omega+1) / 2}}
$$

in which $\overline{\mathcal{N}}$ is the normalization constant, $\bar{b}=2 \sqrt{b \bar{\omega} / \gamma}$, and $K_{\nu}$ is the modified Bessel function of the second kind. In particular, for $\gamma=2$ we note that $P(\ell)$ is the $K$-distribution [54] (see Table I).

Since the Bessel function behaves as $K_{\nu}(x) \sim e^{-x} / \sqrt{x}$ for large- $x$, we observe that the inequalities between the parameters due to the normalization and finite second moment constraints lead the distribution (9) to display only diffusive behavior. However, Lévy-like limited superdiffusive movement dynamics is also possible if $\gamma>0$ and the constraint on $a / \varepsilon$ is relaxed, so that $a / \varepsilon=1+r$ for a specific range of values $0<r<\gamma / 2$.

Interestingly, if, on the other hand, $f(\varepsilon)$ is chosen as an inverse-gamma, we find that the limited superdiffusive behavior cannot take place.

A further generalization is possible when the move lengths in a long path can be grouped into statistically independent subsets associated with different phase dynamics, as, for instance, in composite or intermittent random walk models of animal movement [58-63]. In general terms, distinct phase dynamics can be related, e.g., to an individual moving in a strongly heterogeneous patchy environment consisting of disjoint spatial regions with rather distinct characteristics. Another example may involve different internal rhythms and animal motivations connected with quite different movement tasks (e.g., area-restricted search, dispersal, or homing). In such cases the distribution is expressed as a statistical mixture of subsets of move lengths,

$$
P(\ell)=\sum_{i=1}^{N_{s}} \omega_{i} P_{i}(\ell),
$$

where $\omega_{i}>0$ denotes the statistical weight of the subset $i$, in a total of $N_{s}$ subsets, so that $\sum_{i=1}^{N_{s}} \omega_{i}=1$. As a recent important example, we mention the hyper-exponential distribu- 
tions of move lengths [28, 45, 47],

$$
P(\ell)=\sum_{i=1}^{N_{s}} \frac{\omega_{i}}{\lambda_{i}} e^{-\ell / \lambda_{i}},
$$

combining exponential distributions with diverse decay rates $1 / \lambda_{i}>0$, which has been applied to model the motion of the nematode C. elegans [47], mussels [28], and bumblebees [45].

\section{DISCUSSION AND CONCLUDING REMARKS}

The move length distribution is a key hallmark of animals' dynamical behavior. By considering a statistical physics approach based on the Langevin and superstatistics frameworks, we have calculated in this work a number of distributions of move lengths $P(\ell)$ in situations in which the statistical properties of the noise do not change along the animal's path and also in the case of heterogeneous noise statistics as well. In particular, the latter may be related to heterogeneous landscapes.

Our approach differs markedly from the Ornstein-Uhlenbeck processes [6, 64-67] usually employed to model the animal's velocities or spatial coordinates in a Langevin formalism with additive noise. Here, instead, we have focused on the move length dynamics, in which the action of an additive noise is argued to lead to inconsistency, that we circumvent with the introduction of an $\ell$-dependent (both linear and nonlinear) multiplicative noise.

We notice that the possible distributions $P(\ell)$ are not limited to the cases worked here, since others can be obtained for distinct choices of the deterministic and stochastic properties in the Langevin dynamics. Also, non-Markvovian and fractional Brownian motion approaches can lead to other possibilities as well. In this regard, it is also important to highlight that the unique solution of each specific stochastic differential Langevin equation, using the numerical Milstein method, allows to assess the full-fledged stochastic process, as opposed to just the stationary distribution.

Importantly, the advance in the techniques to register animal movement behavior (such as, e.g., the GPS technology and highly-sensitive motion detection cameras), along with new procedures to segment animal trajectories accurately and the use of MLE and AIC inference methods applied to the statistical analysis of empirical data, can be combined

with our analytical and numerical results to assess and promote hypothesis testing on the type of nonlinearity associated with the deterministic component of movement and the 
stochastic properties influencing the move lengths. In this sense, not only the analysis of the move length distribution is relevant by itself, but also the general dynamical properties of the actual trajectories, such as the rms distance, correlation functions and their power spectrum, which can be compared with the numerical solution of the Langevin dynamics.

The study of the distribution of animal move lengths is also important to the current key discussion about the prevalence of superdiffusive Lévy-like or diffusive composite patterns of movement as the optimal strategy for animals searching for resources in depleted environments $[1,5,6,58-61]$. While diffusive strategies rely on distributions with finite (generally small) number of length scales, Lévy-like power-law models are scale free (or, alternatively, present infinite scales [5]). The scenario that maximizes the search efficiency in depleted landscapes is still an unsettled question in the context of animal motion [68-70].

In addition, it might be also argued [71] that the number of candidates for move length distributions that lead to plastic and overall efficient movement patterns may be not too large. This contrasts with strictly context-dependent situations, where specific local interactions can give rise to far more diverse families of $P(\ell)$. Thus, it is worth investigating in a future work if, besides being related to responses to local stimuli, a given $P(\ell)$ is also influenced by more general selective pressures, like optimization or environment-induced adaptive emergence [1].

In conclusion, we believe the present study may help to guide empirical research and test some potential mechanisms that advance beyond the level of blind fitting to some given distribution of move lengths. We thus hope to stimulate further theoretical and experimental works that improve the overall understanding of the move length distributions in animal motion.

\section{ACKNOWLEDGMENTS}

This work was supported by the Brazilian agencies CNPq, CAPES, and FACEPE through the Pronex project APQ-0602-1.05/14. 


\section{Appendix A: Connection between the Langevin dynamics and Fokker-Planck equa-} tion

We aim in this Appendix to review, for the benefit of the interested reader, the main steps that bridge between the Langevin dynamics and Fokker-Planck equation.

We begin by considering that the dynamics of the variable $\ell(t)$ is given by the Langevin stochastic differential equation (SDE), Eq. (1),

$$
d \ell(t)=F(\ell(t), t) d t+G(\ell(t), t) d W_{t}
$$

in which the function $F$ sets the deterministic part and the stochastic (noise) term is described by a Wiener process, $d W_{t}=\xi(t) d t$, modulated by the function $G$, where $\xi(t)$ has Gaussian distribution (Gaussian white noise), with average $\langle\xi(t)\rangle=0$ and correlation $\left\langle\xi(t) \xi\left(t^{\prime}\right)\right\rangle=2 \varepsilon \delta\left(t-t^{\prime}\right)$, and $\delta$ denotes the Dirac delta function.

By integrating Eq. (A1) in a small time interval $\Delta t$, we find [24]

$$
\ell(t+\Delta t)-\ell(t)=\int_{t}^{t+\Delta t} F d t^{\prime}+\int_{t}^{t+\Delta t} G d W_{t^{\prime}}
$$

After expanding $F$ and $G$ in Taylor series up to first order in $\ell(t+\Delta t)-\ell(t)$, and iterating once by substituting $\ell(t+\Delta t)-\ell(t)$ back into the integrands above, we obtain the average

$$
\langle\ell(t+\Delta t)-\ell(t)\rangle=\int_{t}^{t+\Delta t} F d t^{\prime}+\int_{t}^{t+\Delta t} F^{\prime} d t^{\prime} \int_{t}^{t^{\prime}} F d t^{\prime \prime}
$$

where $F^{\prime}=\partial F / \partial \ell$. Above we have used the average properties of the Gaussian noise and worked under Itô prescription [24], in which the noise variable $\xi$ is evaluated at the beginning of the interval $[t, t+d t)$, so that $\langle G \xi(t)\rangle=0$. From Eq. (A3), we thus find

$$
a_{1}(\ell(t), t)=\lim _{\Delta t \rightarrow 0} \frac{\langle\ell(t+\Delta t)-\ell(t)\rangle}{\Delta t}=F(\ell(t), t) .
$$

In a similar way, we obtain

$$
a_{2}(\ell(t), t)=\lim _{\Delta t \rightarrow 0} \frac{\left\langle[\ell(t+\Delta t)-\ell(t)]^{2}\right\rangle}{\Delta t}=2 \varepsilon[G(\ell(t), t)]^{2} .
$$

The connection of the above results with the Fokker-Planck equation can be found by initially considering the master equation [24],

$$
\frac{\partial P(\ell, t)}{\partial t}=\int\left[W\left(\ell \mid \ell^{\prime}\right) P\left(\ell^{\prime}, t\right)-W\left(\ell^{\prime} \mid \ell\right) P(\ell, t)\right] d \ell^{\prime},
$$


where $P(\ell, t)$ gives the probability density that the variable assumes the value $\ell$ at time $t$, $W\left(\ell \mid \ell^{\prime}\right)$ is the transition probability rate from $\ell^{\prime}$ to $\ell$, and the integral accounts for all possible transitions. Assuming $W\left(\ell \mid \ell^{\prime}\right)=W\left(\ell^{\prime}, y\right)$, with $y=\ell-\ell^{\prime}$, we write

$$
\frac{\partial P(\ell, t)}{\partial t}=\int W(\ell-y, y) P(\ell-y, t) d y-P(\ell, t) \int W(\ell,-y) d y .
$$

Now, by expanding the integrand of the first integral above in Taylor series, we obtain up to order $y^{2}$,

$$
\frac{\partial P(\ell, t)}{\partial t}=-\frac{\partial\left[\alpha_{1}(\ell, t) P(\ell, t)\right]}{\partial \ell}+\frac{1}{2} \frac{\partial^{2}\left\{\alpha_{2}(\ell, t) P(\ell, t)\right\}}{\partial \ell^{2}}
$$

where we identify the moments of the transition probability rate,

$$
\alpha_{n}(\ell(t), t)=\int W(\ell, y) y^{n} d y
$$

Let us now compute the averages $\left\langle[\ell(t+\Delta t)-\ell(t)]^{m}\right\rangle$, with integer $m$, for a small time interval $\Delta t$ :

$$
\left\langle[\ell(t+\Delta t)-\ell(t)]^{m}\right\rangle=\int\left(\ell^{\prime}-\ell\right)^{m} P\left(\ell^{\prime}, t+\Delta t \mid \ell, t\right) d \ell^{\prime} .
$$

By writing the conditional probability to the lowest order in $\Delta t$ in the form

$$
P\left(\ell^{\prime}, t+\Delta t \mid \ell, t\right)=\delta\left(\ell^{\prime}-\ell\right) p(\ell) \Delta t+W\left(\ell^{\prime} \mid \ell\right) \Delta t
$$

where $p(\ell) \Delta t$ gives the probability that no transition $\ell \rightarrow \ell^{\prime}$ occurs during $\Delta t$, and substituting (A11) into (A10), we obtain

$$
\left\langle[\ell(t+\Delta t)-\ell(t)]^{m}\right\rangle=\alpha_{m}(\ell(t), t) \Delta t
$$

where we have used Eq. (A9) and $y=\ell^{\prime}-\ell$. Finally, by comparing Eq. (A12) with Eqs. (A4) and (A5) in the limit $\Delta t \rightarrow 0$, we note that $\alpha_{1}=a_{1}$ and $\alpha_{2}=a_{2}$, which, upon substitution into Eq. (A8), yields the Fokker-Planck equation:

$$
\frac{\partial P(\ell, t)}{\partial t}=-\frac{\partial[F(\ell, t) P(\ell, t)]}{\partial \ell}+\varepsilon \frac{\partial^{2}\left\{[G(\ell, t)]^{2} P(\ell, t)\right\}}{\partial \ell^{2}} .
$$

To illustrate the connection between the Langevin dynamics (1) and the associated Fokker-Planck equation (A13), we consider the deterministic part $F(\ell)$ of (1) as given by Eq. (2) with $c \rightarrow 0^{+}$and the linear multiplicative noise, $G(\ell)=\ell$. The stationary solution $P(\ell)$ can be found by integrating twice Eq. (A13), with $\partial P / \partial t=0$ as $t \rightarrow \infty$, thus leading to the move length distribution in Eq. (3). On the other hand, by taking into account the same function $F(\ell)$ but with nonlinear noise, $G(\ell)=\ell^{s}$, for $s>0$ and $s \neq 1$, then 
distribution (7) is obtained. In both cases, the parameter inequalities are set by requiring normalization to unity, $P(\ell \rightarrow \infty)=0$, and finite second moment of $P(\ell)$, as expected in the context of animal motion.

At this point, we comment that other choices are also possible for the evaluation of the noise variable $\xi$ in the interval $[t, t+d t)$, rather than the Itô one that led to Eq. (A3). For instance, in the Stratonovich prescription [24] $\xi$ is evaluated at the middle of the interval, so that $\langle G \xi(t)\rangle=(G / 2) \partial G / \partial \ell$. This leads to an extra term $-\varepsilon \partial[G P \partial G / \partial \ell] / \partial \ell$ in the right hand side of Eq. (A13). Since $\partial G / \partial \ell \neq 0$ in the case of multiplicative noise, the stationary solutions $P(\ell)$ change under the Stratonovich prescription, if compared with the Itô one. However, we remark that the families of distributions derived in this work remain unaltered. For instance, the only change in Eq. (3) obtained under Itô scheme would be in the power-law exponent $2-a / \varepsilon$, which in the Stratonovich prescription becomes $1-a / \varepsilon$.

\section{Appendix B: The Milstein method}

We now provide the basic information on the Milstein method [49] applied in this work to solve numerically the Langevin equation, Eq. (1).

We start by defining

$$
\omega(h)=W_{t+h}-W_{t}=\int_{t}^{t+h} \xi\left(t^{\prime}\right) d t^{\prime},
$$

where we consider that the time variable $t$ changes by small discretized amounts $\Delta t=h$. Next, by proceeding similarly as in Eq. (A2), we obtain [49]

$$
\ell(t+h)=\ell(t)+F h+G \omega(h)+G G^{\prime} \int_{0}^{\omega(h)} \omega^{\prime} d \omega^{\prime} .
$$

where $G^{\prime}=\partial G / \partial \ell$.

Under Itô prescription, it can be found that [49]

$$
\int_{0}^{\omega(h)} \omega^{\prime} d \omega^{\prime}=\frac{\omega^{2}(h)}{2}-h .
$$

We then apply the average properties of the Gaussian white noise in Eq. (B1), yielding $\langle\omega(h)\rangle=0$ and $\left\langle\omega(h) \omega\left(h^{\prime}\right)\right\rangle=2 \varepsilon h \delta\left(h-h^{\prime}\right)$. Therefore, by substituting Eq. (B3) into Eq. (B2) along with these averages, the numerical simulation of the variable $\ell(t)$ is performed through the dynamics equation

$$
\ell(t+h)=\ell(t)+F h+u G \sqrt{2 \varepsilon h}+\varepsilon G^{\prime} G\left(u^{2}-1\right) h
$$


where $u$ is a random number drawn from a Gaussian distribution with zero mean and unit variance. Here we notice that after $N_{t}=10^{6}$ iterations of Eq. (B4) using $h=10^{-3}$, i.e., after a time $t=N_{t} h=10^{3}$, the stationary regime of the Langevin dynamics (1) is fairly reached (i.e., the distribution of move lengths becomes $t$-independent), and then the move length $\ell$ is recorded. In order to build each move length distribution $P(\ell)$ shown in Fig. 1, this whole process was repeated $10^{8}$ times so to generate an ensemble of $10^{8}$ values of $\ell$.

In the numerical simulation of a random walk particle in one dimension with move length distribution $P(\ell)$, whose rms distance is shown in Fig. 2 as a function of the number of moves $N$, we performed averages over $10^{6}$ walk realizations up to $N=10^{3}$, when fluctuations in the random walker's position are stronger, and over $10^{4}$ realizations for larger $N$.

[1] Nathan R ed 2008 Movement Ecology Special Feature. Proc. Natl. Acad. Sci. USA 10519050

[2] Swingland I R and Greenwood P J eds 1983 The Ecology of Animal Movement (Oxford: Oxford Univ Press)

[3] Turching P 1998 Quantitative Analysis of Movement (Sunderland-MA: Sinauer)

[4] Hansson L-A and Akesson S 2014 Animal Movement Across Scales (Oxford: Oxford Univ Press)

[5] Viswanathan G M, da Luz M G E, Raposo E P and Stanley H E 2011 The Physics of Foraging (Cambridge: Cambridge Univ Press)

[6] Méndez V, Campos D and Bartumeus F 2014 Stochastic Foundations in Movement Ecology (New York: Springer)

[7] Nathan R et al 2008 Proc. Natl. Acad. Sci. USA 10519052

[8] Getz W M and Saltz D 2008 Proc. Natl. Acad. Sci. USA 10519066

[9] Bartumeus F and Levin S A 2008 Proc. Natl. Acad. Sci. USA 10519072

[10] Lenz F, Chechkin A V and Klages R 2013 PLoS ONE 8 e59036

[11] Smouse P E et al 2010 Philos. Trans. R. Soc. B 3652201

[12] Gomez-Marin A, Paton J J, Kampff A R, Costa R M and Mainen Z F 2014 Nat. Neurosci. 171455

[13] Humphries N E, Weimerskirch H and Sims D W 2013 Methods Ecol. Evol. 4930

[14] Edelhoff H, Signer J and Balkenhol N 2016 Mov. Ecol. 41 
[15] Demsar U et al 2015 Mov. Ecol. 31

[16] Patterson T A et al 2017 Adv. Stat. Analys. 101399

[17] Bastille-Rousseau G et al 2016 Mov. Ecol. 415

[18] Morales J M, Haydon D T, Frair J, Holsinger K E and Fryxell J M 2004 Ecology 852436

[19] McClintock B T, King R, Thomas L, Matthiopoulos J, McConnell B J and Morales J M 2012 Ecol. Monogr. 82335

[20] Viswanathan G M et al 1999 Nature 401911

[21] Namboodiri V M K, Levy J M, Mihalas S, Sims D W and Shuler M G H 2016 Proc. Natl. Acad. Sci. USA 1138747

[22] Da Luz M G E, Raposo E P and Viswanathan G M 2016 Proc. Natl. Acad. Sci. USA 113 8571

[23] Bartumeus F, Raposo E P, Viswanathan G M, da Luz M G E 2014 PLoS ONE 9 e106373

[24] Wilkinson D J 2011 Stochastic Modelling for Systems Biology (Boca Raton-FL: CRC Press)

[25] Beck C and Cohen E 2003 Physica A 322267

[26] Metzner C et al 2015 Nat. Commun. 67516

[27] Torney C J, Hopcraft J G C, Morrison T A, Couzin I D and Levin S A 2018 Phil. Trans. R. Soc. B 37320170012

[28] Reynolds A M 2014 Sci. Rep. 44409

[29] Mandelbrot B, van Ness J W 1968 SIAM Rev. 10422

[30] Samorodnitsky G, Taqqu M S 1994 Stable Non-Gaussian Random Processes (New York: Chapman Hall)

[31] Magdziarz M, Weron A, Burnecki K, Klafter J 2009 Phys. Rev. Lett. 103180602

[32] Wada A H O, Vojta T 2018 Phys. Rev. E 97020102

[33] Giuggioli L, McKetterick T J, Kenkre V M, Chase M 2016 J. Phys. A 49384002

[34] Lutz E 2001 Phys. Rev. E 64051106

[35] Pottier N 2003 Physica A 317371

[36] Burov S and Barkai E 2008 Phys. Rev. Lett. 100070601

[37] Täuber U C 2014 Critical Dynamics: A Field Theory Approach to Equilibrium and NonEquilibrium Scaling Behavior (Cambridge: Cambridge Univ Press)

[38] Van Kampen N G 2007 Stochastic Processes in Physics and Chemistry (Amsterdam: Elsevier)

[39] Kamenev A, Meerson B, Shklovskii B 2008 Phys. Rev. Lett. 101268103 
[40] Cox D R, Miller H D 1965 The Theory of Stochastic Processes (Boca Raton-FL: Chapman \& Hall)

[41] Hongler M-O, Filliger R 2019 Methodol. Comput. Appl. Prob. 21753

[42] Codling E A, Plank M J and Benhamou S 2008 J. R. Soc. Interface 5813

[43] Sims D W et al 2008 Nature 4511098

[44] Humphries N E et al 2010 Nature 4651066

[45] Kembro J M, Lihoreau M, Garriga J, Raposo E P and Bartumeus F 2019 J. R. Soc. Interface 1620190103.

[46] Ramos-Fernández G et al 2004 Behav. Ecol. Sociobiol. 55223

[47] Bartumeus F et al 2016 Ecol. Lett. 191299

[48] Edwards A M et al 2007 Nature 4491044.

[49] Kloeden P E and Platen E 2013 Numerical Solution of Stochastic Differential Equations (Berlin: Springer)

[50] Reynolds A M 2014 Proc. R. Soc. A 47020140408

[51] Kolzsch A et al 2015 Proc. R. Soc. B 28220150424

[52] Mantegna R N and Stanley H E 1994 Phys. Rev. Lett. 732946

[53] Jørgensen B 1982 in Lecture Notes in Statistics 9 (New York: Springer-Verlag).

[54] Panik M J 2005 Advanced Statistics from an Elementary Point of View (San Diego: Elsevier).

[55] Dalziel B D, Morales J M and Fryxell J 2008 Am. Nat. 172248

[56] Salazar D S P and Vasconcelos G L 2010 Phys. Rev. E 82047301

[57] Macêdo A M S, González I R R, Salazar D S P and Vasconcelos G L 2017 Phys. Rev. E 95 032315

[58] Benhamou S 2007 Ecology 881962

[59] Lomholt M A, Koren T, Metzler R and Klafter J 2008 Proc. Natl. Acad. Sci. USA 10511055

[60] Reynolds A 2006 EPL 75517

[61] Bénichou O, Loverdo C, Moreau M and Voituriez R 2011 Rev. Mod. Phys. 8381

[62] O'Brien W J, Browman H I and Evans B I 1990 Am. Sci. 78152

[63] Kramer L D and McLaughlin R L 2001 Amer. Zool. 41137

[64] Niu M, Blackwell P G and Skarin A 2016 Biometrics 72315

[65] McClintock B T, Johnson D S, Hooten M B, Ver Hoef J M and Morales J M 2014 Mov. Ecol. 221 
[66] Patterson T A et al 2017 Adv. Stat. Anal. 101399

[67] Gurarie E, Fleming C H, Fagan W F, Laidre K L, Hernández-Pliego J and Ovaskainen O 2017 Mov. Ecol. 513

[68] Reynolds A 2015 Phys. Life Rev. 1459

[69] Da Luz M G E, Raposo E P and Viswanathan G M 2015 Phys. Life Rev. 1494

[70] Bartumeus F 2015 Phys. Life Rev. 1484

[71] Wosniack M E, Santos M C, Raposo E P, Viswanathan G M and da Luz M G E 2017 PLoS Comp. Biol. 13 e1005774 